PROCEEDINGS OF THE

AMERICAN MATHEMATICAL SOCIETY

Volume 134, Number 11, November 2006, Pages 3379-3390

S 0002-9939(06)08543-1

Article electronically published on May 8, 2006

\title{
A FAMILY OF MAXIMAL SURFACES IN LORENTZ-MINKOWSKI THREE-SPACE
}

\author{
YOUNG WOOK KIM AND SEONG-DEOG YANG
}

(Communicated by Richard A. Wentworth)

\begin{abstract}
We prove the existence of an infinite family of complete spacelike maximal surfaces with singularities in Lorentz-Minkowski three-space and study their properties. These surfaces are distinguished by their number of handles and have two elliptic catenoidal ends.
\end{abstract}

\section{INTRODUCTION}

Spacelike maximal surfaces in the Lorentz-Minkowski three-space $\mathbb{L}^{3}$ arise as solutions of the variational problem of locally maximizing the area among spacelike surfaces. By definition, they have everywhere vanishing mean curvature. There are many interesting local properties of maximal surfaces similar to those of minimal surfaces. In particular, maximal surfaces possess a Weierstrass-type representation formula 12 .

The most significant difference between minimal and maximal surfaces is the existence of singularities for maximal spacelike surfaces. It has been known that spacelike planes are the only spacelike maximal surfaces which are complete [2, 3], which is probably the main reason why people have not paid much attention to maximal surfaces, and there are not so many known examples of interesting maximal surfaces.

If we allow some sort of singularities for maximal surfaces, however, the situation changes. Recently many interesting examples with isolated singularities have been found and studied by F. J. López, R. López, Fernández, and Souam [5, 6, 7, 14]. Umehara and Yamada showed that if admissible singularities are included, then there is an interesting class of objects which they call maxfaces [17].

In this article, we show that there exists an infinite family of maximal surfaces with singularities (Figure 1) and investigate their properties. Our main results are

Theorem 1 (Main Theorem). For every natural number $k$, there exists a maximal surface with singularities, which we call $\mathcal{M}_{k}$, with the following properties:

(i) $\mathcal{M}_{k}$ is parameterized on a compact Riemann surface of genus $k$ with two points removed.

Received by the editors April 18, 2005 and, in revised form, May 23, 2005.

2000 Mathematics Subject Classification. Primary 53A10, 53C50.

Key words and phrases. Lorentz-Minkowski space, spacelike maximal surface, elliptic catenoidal ends.

(C)2006 American Mathematical Society Reverts to public domain 28 years from publication 3379 

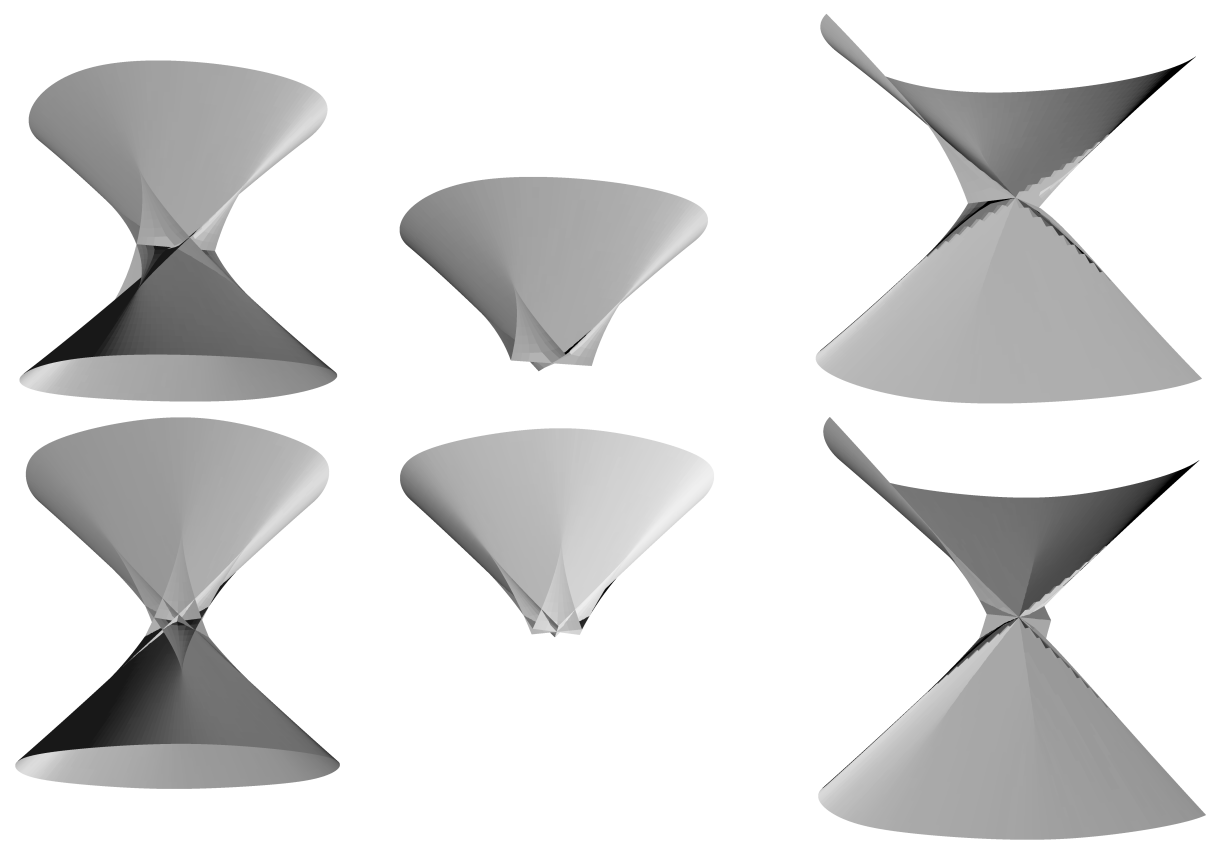

Figure 1. Genus 1 and 2 examples, their upper halves and vertical slices

(ii) $\mathcal{M}_{k}$ is a complete generalized maximal surface [4. In particular, $\mathcal{M}_{1}$ is a complete maxface of finite type [17.

(iii) $\mathcal{M}_{k}$ has two ends, both of which are elliptic catenoidal.

(iv) $\mathcal{M}_{k}$ is not embedded.

(v) $\mathcal{M}_{k}$ intersects the xy-plane in $k+1$ quadruple line segments (of finite length), which meet at equal angles at the origin. Removal of the line segments disconnects the maximal surface into a union of two topological annuli.

(vi) There are $4(k+1)$ swallowtail singularities. All the other singularities except the ones in the xy-plane are cuspidal edges. The singularities in the xy-plane are cuspidal crosscaps.

(vii) $\mathcal{M}_{k}$ has a symmetry group of order $8(k+1)$, which are generated by

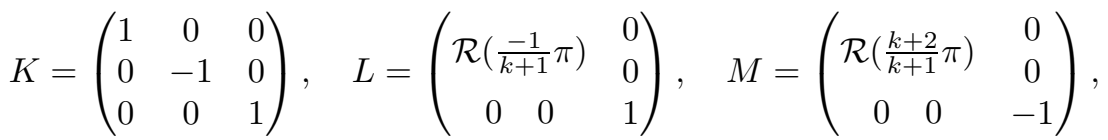

where $\mathcal{R}(\theta)$ is the matrix of rotation by $\theta$ in the $x y$-plane.

According to Schoen [16], catenoids in $\mathbb{E}^{3}$ are the only complete minimal surfaces of finite total curvature with 2 embedded ends. Existence of $\mathcal{M}_{1}$ shows that Schoen's nonexistence result does not hold for complete maximal surfaces with singularities of finite type, with the definition of being complete and of finite type as in Definition 3, It should be remarked that the integral of the Gauss curvature on $\mathcal{M}_{k}$ outside the singular set is infinite. Rossman and Sato previously showed that there exists a genus 1 immersed catenoid in $\mathbb{H}^{3}(-1)[15$. 
The method of construction is to use the Weierstrass representation theorem. The Weierstrass data as well as the main ideas for the existence proof have been motivated by the work of Hoffman and Meeks III [9], and the idea is explained in 18. In view of the ways in which Costa-Hoffman-Meeks family of minimal surfaces are deformed, it is expected that these maximal surfaces can be deformed in various ways.

We thank Fujimori, Rossman, Umehara and Yamada for many helpful conversations as well as pointing out an error in an earlier version of this article, and Jae Ung $\mathrm{Yu}$ for drawing the pictures in this article.

\section{Preliminaries}

In this article, the coordinates system of the Lorentz-Minkowski three-space $\mathbb{L}^{3}$ is set as follows:

$$
\mathbb{L}^{3}=\left\{(x, y, t) \in \mathbb{R}^{3}: d s^{2}=d x^{2}+d y^{2}-d t^{2}\right\} .
$$

An immersion $X: M^{2} \rightarrow \mathbb{L}^{3}$ of a 2 -manifold is called spacelike if the induced metric is positive definite. A spacelike immersion is called maximal if the mean curvature is 0 everywhere. A maximal map is a smooth map from a 2-manifold into $\mathbb{L}^{3}$ if there is an open dense subset $W$ of $M^{2}$ such that $\left.f\right|_{W}$ is a maximal immersion. A maxface is defined to be a maximal map all of whose singular points are admissible. See [17] for details. It is in this category that we state the following Weierstrass representation formula for maximal spacelike surfaces.

Theorem 2 ([12, 17]). Let $g$ and $\eta$ be a meromorphic function and a holomorphic 1-form on a Riemann surface $M^{2}$ such that $\left(1+|g|^{2}\right)^{2}|\eta|^{2}$ is a Riemannian metric on $M^{2}$ and $\left(1-|g|^{2}\right)^{2}|\eta|^{2}$ does not vanish identically. Then the following defines a maxface from $M^{2}$ to $\mathbb{L}^{3}$ :

$$
X: M^{2} \rightarrow \mathbb{L}^{3}, \quad X(p)=\operatorname{Re} \int_{p_{0}}^{p}\left(\left(1+g^{2}\right) \eta, i\left(1-g^{2}\right) \eta, 2 g \eta\right)
$$

if all the periods vanish. Furthermore,

(i) the Gauss map $\nu$ is given by

$$
\nu=\left(\frac{2 \operatorname{Re} g}{1-|g|^{2}}, \frac{2 \operatorname{Im} g}{1-|g|^{2}}, \frac{1+|g|^{2}}{1-|g|^{2}}\right),
$$

(ii) the induced metric is $d s^{2}=\left(1-|g|^{2}\right)^{2}|\eta|^{2}$,

(iii) the Gauss curvature of the surface is $K=\frac{4}{\left(1-|g|^{2}\right)^{4}} \frac{|d g|^{2}}{|\eta|^{2}}$.

The converse also holds.

The simplest examples of maximal surfaces with singularities are the rotationally invariant maximal surfaces. There are three kinds of such surfaces depending upon the causal character of the axis of rotation [1] (Figure 21).

Definition $3([17])$. A maxface $X: M^{2} \rightarrow \mathbb{L}^{3}$ is complete (resp. of finite type) if there exist a compact set $C \subset M^{2}$ and a symmetric 2-tensor $T$ on $M^{2}$ such that $T$ vanishes on $M^{2} \backslash C$ and $d s^{2}+T$ is a complete metric (resp. a metric of finite total Gaussian curvature) on $M^{2}$, where $d s^{2}$ is the pull-back of the Lorentz-Minkowski metric by $X$. 

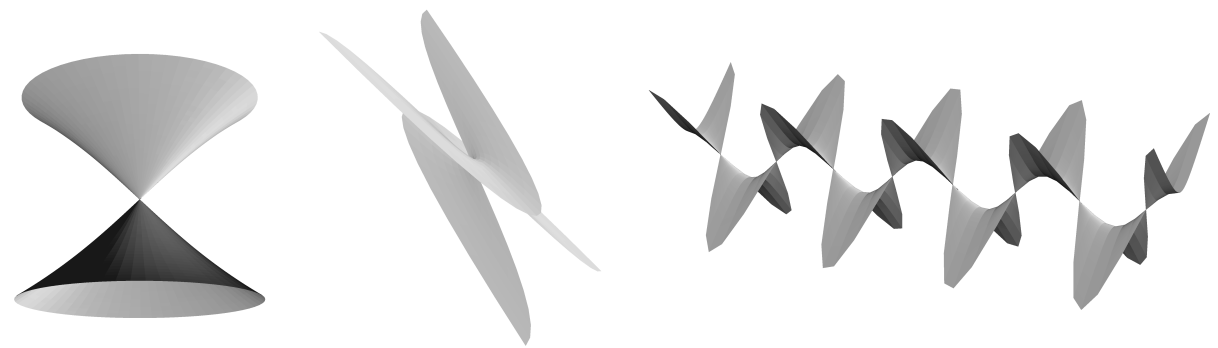

FIGURE 2. Elliptic, parabolic, and hyperbolic catenoids

We use $x, y, t: M^{2} \rightarrow \mathbb{R}$ to denote the coordinate functions. Then, it is immediate from (2) that

$$
(x+i y)(p)=\overline{\int_{p_{0}}^{p} \eta}+\int_{p_{0}}^{p} g^{2} \eta, \quad t(p)=\int_{p_{0}}^{p} g \eta+\overline{\int_{p_{0}}^{p} g \eta} .
$$

\section{The Weierstrass Data}

Let $k \in\{1,2,3, \cdots\}$ be an arbitrary natural number. Consider the closed Riemann surface of genus $k$,

$$
\bar{M}_{k}=\left\{(\alpha, \beta) \in(\mathbb{C} \cup\{\infty\})^{2}: \beta^{k+1}=\alpha^{k}(\alpha+1)(\alpha-1)\right\} .
$$

The maximal surfaces we construct have the following Weierstrass data:

$$
\eta=\frac{d \alpha}{\beta}, \quad g=\sigma \frac{\beta}{\alpha}, \quad M_{k}=\bar{M}_{k} \backslash\{(0,0),(\infty, \infty)\}, \quad\left(\alpha_{0}, \beta_{0}\right)=(1,0),
$$

where $\sigma$ is a positive real number to be determined later. $\left(\alpha_{0}, \beta_{0}\right)=(1,0)$ is the reference point for the integration of the associated one-forms in the Weierstrass representation theorem, hence the image of $\left(\alpha_{0}, \beta_{0}\right)=(1,0)$ is the origin $(0,0,0) \in$ $\mathbb{L}^{3}$.

\section{Symmetries of the Weierstrass data}

Definition 4. Let $c:=e^{\frac{\pi}{k+1} i}$, and let $\kappa, \lambda, \mu:(\mathbb{C} \cup\{\infty\})^{2} \rightarrow(\mathbb{C} \cup\{\infty\})^{2}$ be

$$
\kappa(\alpha, \beta):=(\bar{\alpha}, \bar{\beta}), \quad \lambda(\alpha, \beta):=\left(-\alpha, c^{k} \beta\right), \quad \mu(\alpha, \beta):=\left(\alpha^{-1}, c \alpha^{-2} \beta\right) .
$$

They preserve $\bar{M}_{k}$. In the rest of this article, we think of them as defined on $\bar{M}_{k}$. Note that $\lambda$ does not preserve the base point of integration $(1,0) \in M_{k}$, unlike $\kappa$ and $\mu$. It is straightforward to see that the order of $\kappa$ is 2, that the order of $\lambda$ and $\mu$ is $2(k+1)$, and that $\kappa, \tau, \mu$ form a group of order $8(k+1)$. We will see later that these conformal diffeomorphisms induce the symmetries of the resulting maximal surfaces. As a first step to observe it, we prove

Lemma 5. $\kappa, \lambda, \mu$ preserve $M_{k}$. Furthermore,

$$
\begin{aligned}
g \circ \kappa & =\bar{g}, & g \circ \lambda & =-c^{k} g, & g \circ \mu & =c g, \\
\kappa^{*} \eta & =\bar{\eta}, & \lambda^{*} \eta & =-c^{-k} \eta, & \mu^{*} \eta & =-c^{-1} \eta .
\end{aligned}
$$



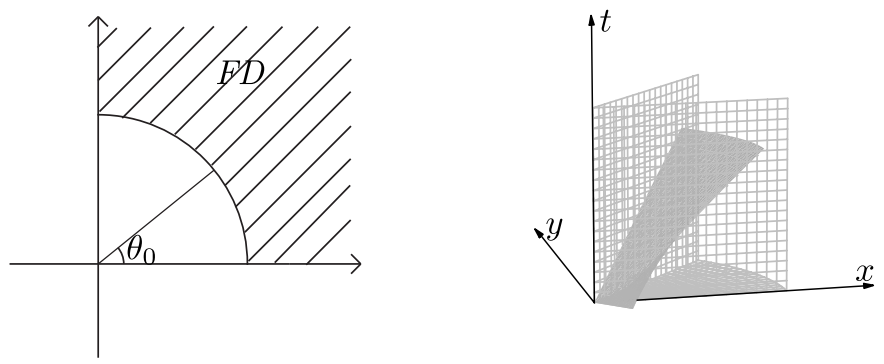

Figure 3 . FD and its image by $X$

The proof is straightforward. An immediate consequence is the following.

Corollary 6. Let

$$
\phi=\left(\phi_{1}, \phi_{2}, \phi_{0}\right)^{T}=\left(\left(1+g^{2}\right) \eta, i\left(1-g^{2}\right) \eta, 2 g \eta\right)^{T} .
$$

Then

$$
\kappa^{*} \phi=K \bar{\phi}, \quad \lambda^{*} \phi=L \phi, \quad \mu^{*} \phi=M \phi,
$$

where $K, L, M$ are the matrices in (1).

\section{Resolution of the PERIOD PROBlem}

For convenience, we sometimes regard $M_{k}$ as obtained by gluing $k+1$ number of $\alpha$-planes with the branch cuts between $\infty$ and -1 and between 0 and 1 along the real line. We fix once and for all the $\alpha$-plane with $\beta(\alpha)>0$ for $\alpha>1$, and a set $F D$ in this $\alpha$-plane (Figure 3 ):

$$
F D:=\left\{\alpha \in \mathbb{C}: 0 \leq \arg \alpha \leq \frac{\pi}{2},|\alpha| \geq 1\right\} .
$$

$8(k+1)$ copies of $F D$ are needed to cover $M_{k}$ through the action of $\kappa, \tau$, and $\mu$.

Let $\gamma(t)=\frac{1}{2}+e^{i t}, 0 \leq t \leq 2 \pi$. Then, a homology basis of $\bar{M}_{k}$ is generated by applying $\kappa$ and $\mu$ to $\gamma$. Therefore, to show that the immersion $X$ is well defined on $M_{k}$, we only need to check whether the periods on $\gamma$ and on loops around $(0,0),(\infty, \infty)$ vanish.

Lemma 7. The periods around $(0,0)$ and $(\infty, \infty)$ vanish.

Proof. Argue as in the proof of [9, Proposition 3.4] with $\kappa, \mu$ and $K, M$.

To deal with the period around $\gamma$, we first observe from (3) and (4) that

$$
t(\alpha, \beta)=2 \sigma \ln |\alpha|,
$$

which immediately implies that the $t$-component of the period around $\gamma$ vanishes. Now we show the following:

Lemma 8. The xy-components of the period around $\gamma$ vanish if and only if

$$
\sigma=\sqrt{\frac{1}{2} \frac{A}{B}}, \quad \text { where } A=\int_{0}^{1} \frac{d t}{\sqrt[k+1]{t^{k}\left(1-t^{2}\right)}}, \quad B=\int_{0}^{1} \frac{\sqrt[k+1]{t^{k}\left(1-t^{2}\right)} d t}{1-t^{2}} .
$$


Proof. From (3), it follows that the $x y$-components of the period around $\gamma$ vanish if and only if $\overline{\int_{\gamma} \eta}+\int_{\gamma} g^{2} \eta=0$. We will compute each of the integrals as $\gamma$ collapses to the double line segment connecting 1 and 0 . We immediately see that $\int_{\gamma} \eta=$ $\left(c-c^{-1}\right) A$. The situation for $\int_{\gamma} g^{2} \eta=\sigma^{2} \int_{\gamma} \frac{\beta}{\alpha^{2}} d \alpha$ is a bit different since $\int_{c_{\epsilon}} \frac{\beta}{\alpha^{2}} d \alpha$ does not converge as $\epsilon \rightarrow 0$, where $c_{\epsilon}$ is a circle of radius $\epsilon$ centered at 0 . We observe, however, that

$$
\frac{\beta}{\alpha^{2}} d \alpha=-(k+1) d\left(\frac{\beta}{\alpha}\right)+\frac{2 \beta}{\alpha^{2}-1} d \alpha .
$$

Since $\int_{\gamma} \frac{2 \beta}{\alpha^{2}-1} d \alpha=2(c-\bar{c}) B$, we get the conclusion.

Therefore the map $X: M_{k} \rightarrow \mathbb{L}^{3}$ is well defined.

\section{Geometry of the surfaces}

6.1. Symmetries of $X\left(M_{k}\right)$ generated by $K, L, M$. Let $K, L, M$ be the matrices in (11). Recall that $\lambda$ does not preserve the base point of integration, hence $L$ may not be a symmetry of $X\left(M_{k}\right)$. However, we have

Lemma 9. $X(-1,0)=(0,0,0)$.

Proof. Let $\gamma(\theta)=e^{i \theta}$ for $\theta \in[0, \pi]$. Then $(x+i y)(-1,0)=\overline{\int_{\gamma} \eta}+\int_{\gamma} g^{2} \eta$. We evaluate these integrals by collapsing $\gamma$ to the line segment from $(1,0)$ to $(-1,0)$. We immediately see that $\int_{\gamma} \eta=(1-\bar{c}) A$ and that, using ([6) $), \int_{\gamma} g^{2} \eta=(c-1) 2 \sigma^{2} B$. Here $A, B$ are the constants in the statement of Lemma 8 . Therefore, $(x+i y)(-1,0)=0$. It is easy to see that $t(-1,0)=0$.

Then, the following is now immediate.

Lemma 10. (i) $X\left(M_{k}\right)$ is not embedded.

(ii) $L$ is also a symmetry of $X\left(M_{k}\right)$, hence the group of order $8(k+1)$ generated by $K, L, M$ is contained in the symmetry group of $X\left(M_{k}\right)$.

(iii) $M K$ is the reflectional symmetry with respect to the line $y=\tan \left(\frac{-k}{k+1} \frac{\pi}{2}\right) x$, $t=0$.

(iv) $K L$ is the reflectional symmetry with respect to the plane $y=\tan \left(\frac{1}{k+1} \frac{\pi}{2}\right) x$.

(v) $K$ is the reflectional symmetry with respect to the $x$-plane.

(vi) $L^{k-1} K M K L$ is the reflectional symmetry with respect to the xy-plane. (Note, however, that the xy-plane does not intersect $X\left(M_{k}\right)$ orthogonally.)

6.2. Image of the boundary of the fundamental domain. Now let us look at $X(\partial F D)$. Let us define

$$
\gamma_{1}(t):=t \text { and } \gamma_{3}(t):=i t \quad \text { for } 1<t<\infty, \quad \gamma_{2}(\theta):=e^{i \theta} \quad \text { for } 0 \leq \theta \leq \frac{\pi}{2} .
$$

We do not distinguish $\gamma_{i}$ from its image in $F D$. Then, $\partial F D=\gamma_{1} \cup \gamma_{2} \cup \gamma_{3}$. First, let us take a look at $X\left(\gamma_{2}\right)$.

Lemma 11. $t\left(\gamma_{2}(\theta)\right)=0$, and $(x+i y)\left(\gamma_{2}(\theta)\right)=F(\theta) e^{\frac{-k}{k+1} \frac{\pi}{2} i}$ for some nonnegative real valued function $F$ with $F(0)=F\left(\frac{\pi}{2}\right)=0$, which is strictly increasing on $\left[0, \theta_{0}\right]$ and strictly decreasing on $\left[\theta_{0}, \frac{\pi}{2}\right]$, where $\theta_{0}=\arcsin \frac{1}{2 \sigma^{k+1}}$. In particular, $X(i)=X\left(\gamma_{2}\left(\frac{\pi}{2}\right)\right)=(0,0,0)$. 
Proof. Given $\theta \in[0, \pi]$, let $\gamma(t)=e^{i t}, t \in[0, \theta]$. Then,

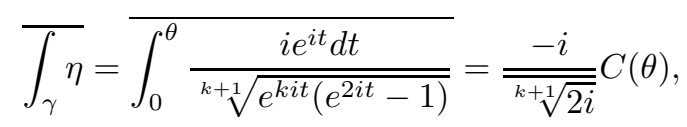

where $C(\theta):=\int_{0}^{\theta} \frac{1}{\sqrt[k+1]{\sin t}} d t$. On the other hand,

$$
\int_{\gamma} g^{2} \eta=\sigma^{2} \int_{0}^{\theta} \frac{\sqrt[k+1]{e^{i(k+1) \theta}\left(e^{i \theta}-e^{-i \theta}\right)}}{e^{2 i \theta}} i e^{i \theta} d \theta=i \sqrt[k+1]{2 i} \sigma^{2} D(\theta)
$$

where $D(\theta)=\int_{0}^{\theta} \sqrt[k+1]{\sin t} d t$. So, if $f(\theta):=C(\theta)-\sqrt[k+1]{4} \sigma^{2} D(\theta)$, then

$$
(x+i y)\left(e^{i \theta}\right)=\frac{-i}{\sqrt[k+1]{2 i}} f(\theta)=\frac{1}{\sqrt[k+1]{2}} e^{-\frac{k}{k+1} \frac{\pi}{2} i} f(\theta) .
$$

We immediately see that $f$ is real-valued and $f(0)=0$. From Lemma 9, we know that $f(\pi)=0$. Since $2 f\left(\frac{\pi}{2}\right)=f(\pi)$ we also have $f\left(\frac{\pi}{2}\right)=0$. We also see that $f^{\prime}(\theta)>0$ if $0<\theta<\theta_{0}$, and $f^{\prime}(\theta)<0$ if $\theta_{0}<\theta<\frac{\pi}{2}$. By letting $F(\theta)=f(\theta) / \sqrt[k+1]{2}$, we have the conclusion.

Hence, $X\left(\gamma_{2}\right)$ is a double line segment in the $x y$-plane. Next we show that $X\left(\gamma_{1}\right)$ and $X\left(\gamma_{3}\right)$ are curves in vertical planes.

Lemma 12. (i) $X\left(\gamma_{1}\right)$ lies in the quarter plane with $x>0, y=0, t>0$, and is the graph of a strictly increasing function $t=t(x)$ with $t(0)=0$.

(ii) $X\left(\gamma_{3}\right)$ lies in the quarter plane $x>0, y=\tan \left(\frac{1}{k+1} \frac{\pi}{2}\right) x, t>0$, and is the graph of a strictly increasing function $t=t\left(\sqrt{x^{2}+y^{2}}\right)$ with $t(0)=0$.

Proof. For $X\left(\gamma_{1}\right)$, we observe that

$$
\begin{aligned}
(x+i y)(u) & =\overline{\int_{1}^{u} \eta}+\int_{1}^{u} g^{2} \eta \\
& =\int_{1}^{u} \frac{d t}{\sqrt[k+1]{t^{k}\left(t^{2}-1\right)}}+\sigma^{2} \int_{1}^{u} \frac{\sqrt[k+1]{t^{k}\left(t^{2}-1\right)}}{t^{2}} d t:=g(u) .
\end{aligned}
$$

Since $g(u) \in \mathbb{R}^{+}$for $u>1$, we have $x(u)>0, y(u)=0$. Since $t(u)=2 \sigma \ln u>0$, we see that $X\left(\gamma_{1}\right)$ lies is the quarter plane stated in the lemma. Furthermore, since $g(1)=0$ and $g^{\prime}(u)>0$ for $u>1$, we see that $x$ is a strictly increasing function of $u$, hence we may regard $u$ as a strictly increasing function of $x$. The conclusion for $X\left(\gamma_{1}\right)$ follows.

For $X\left(\gamma_{3}\right)$, we observe that

$$
\begin{aligned}
(x+i y)(i v) & =\overline{\int_{i}^{i v}} \eta+\int_{i}^{i v} g^{2} \eta \\
& =e^{\frac{1}{k+1} \frac{\pi}{2} i}\left(\int_{1}^{v} \frac{d t}{\sqrt[k+1]{t^{k}\left(t^{2}+1\right)}}+\sigma^{2} \int_{1}^{v} \frac{\sqrt[k+1]{t^{k}\left(t^{2}+1\right)}}{t^{2}} d t\right) .
\end{aligned}
$$

Here we used the fact that $i$ can also be used as the reference point of integration since the image of $\alpha=i$ is also the origin, as was proved in Lemma 11. The conclusion follows in similar ways as above. 
The above lemma along with the following shows that the image by $X$ of a neighborhood of $\gamma_{1}$ and of $\gamma_{3}$ is indeed perpendicular to vertical planes $y=0$ and $y=\tan \left(\frac{1}{k+1} \frac{\pi}{2}\right) x$, respectively. Note that $\eta d g=\frac{\sigma}{k+1} \frac{\alpha+\alpha^{-1}}{\alpha-\alpha^{-1}}\left(\frac{d \alpha}{\alpha}\right)^{2}$ is real on $\gamma_{1}$ and $\gamma_{3}$, and purely imaginary on $\gamma_{2}$.

Lemma 13 (Gauss map on $X(\partial F D)$ ). The unit timelike normal vectors of $X\left(M_{k}\right)$ along $\gamma_{1}$ lie in the vertical plane $y=0$. The unit timelike normal vectors of $X\left(M_{k}\right)$ along $\gamma_{2}$ and on $\gamma_{3}$ lie in the vertical plane $y=\tan \left(\frac{1}{k+1} \frac{\pi}{2}\right) x$.

Proof. We have

$$
\begin{array}{ll}
g \circ \gamma_{1}(t)=g(t)=\sigma \sqrt[k+1]{t-t^{-1}} & \text { for } t \geq 1 \\
g \circ \gamma_{2}(\theta)=g\left(e^{i \theta}\right)=\sigma \sqrt[k+1]{2 \sin \theta} e^{\frac{1}{k+1} \frac{\pi}{2} i} \quad \text { for } \theta \in[0, \pi / 2] & \\
g \circ \gamma_{3}(t)=g(i t)=\sigma \sqrt[k+1]{t+t^{-1}} e^{\frac{1}{k+1} \frac{\pi}{2} i} \quad \text { for } t \geq 1
\end{array}
$$

which imply the claims.

6.3. Asymptotic behavior of the ends. Define $z$ by $\alpha=z^{-(k+1)}$. Then, $z$ in a neighborhood of 0 is a local coordinate for the end $(\alpha, \beta)=(\infty, \infty)$. Using this, we see that

$$
\begin{aligned}
\left(\left(1+g^{2}\right)\right. & \left., i\left(1-g^{2}\right), 2 g\right) \eta \\
& =\left(-\frac{\sigma^{2}(k+1)}{z^{2}}+\mathcal{O}(z), i \frac{\sigma^{2}(k+1)}{z^{2}}+\mathcal{O}(z), \frac{-2 \sigma(k+1)}{z}+\mathcal{O}(z)\right) d z .
\end{aligned}
$$

Therefore, $z=0$, or equivalently $(\alpha, \beta)=(\infty, \infty)$, is a simple end of type $\mathrm{I}$ in the language of [11, which converges to an elliptic catenoid.

6.4. Singularities of the surfaces. Note that, for any $M_{k}$, the metric $d s^{2}$ vanishes at the points where $|g|=\left|\sigma \frac{\beta}{\alpha}\right|=1$. Excluding those points, the metric does not vanish on $M_{1}$, but it vanishes at $(\alpha, \beta)=( \pm 1,0)$ on $M_{k}$ with $k \geq 2$.

We now want to locate the points with $|g|=1$ on $F D . \quad\left|\sigma \frac{\beta}{\alpha}\right|=1$ implies $|\alpha|^{k+1}=\sigma^{k+1}\left|\beta^{k+1}\right|=\sigma^{k+1}|\alpha|^{k}\left|\alpha^{2}-1\right|$, hence $\left|\alpha-\alpha^{-1}\right|=\sigma^{-(k+1)}$. By letting $\alpha=r e^{i \theta}$, this equals

$$
r^{2}+r^{-2}=\sigma^{-2(k+1)}+2 \cos 2 \theta:=t(\theta) .
$$

Since $r^{2}+r^{-2} \geq 2$ for any $r>0$, we conclude that $t \geq 2$. Now

$$
t(\theta) \geq 2 \Leftrightarrow 0 \leq \theta \leq \theta_{0}, \quad \text { where } \theta_{0}=\arcsin \frac{1}{2 \sigma^{k+1}} .
$$

Note that this $\theta_{0}$ has already appeared in Lemma 11. The above shows that the singularities are in $F D \cap\left\{0 \leq \arg \alpha \leq \theta_{0}\right\}$ (Figure 44). For each $\theta \in\left[0, \theta_{0}\right]$, there is a unique value of $r \geq 1$ which solves (7): $r(\theta)=\sqrt{\frac{t(\theta)+\sqrt{t(\theta)^{2}-4}}{2}}$.

Fujimori, Saji, Umehara and Yamada showed that the generic singularities of maximal surfaces in $\mathbb{L}^{3}$ consist of cuspidal edges, swallowtails, and cuspidal crosscaps, whose typical examples are given by $\left(u^{2}, u^{3}, v\right),\left(3 u^{4}+u^{2} v, 4 u^{3}+2 u v, v\right)$, and $\left(u, v^{2}, u v^{3}\right)$, respectively [8, 17]. They cover all the singularities of $X\left(M_{k}\right)$. 


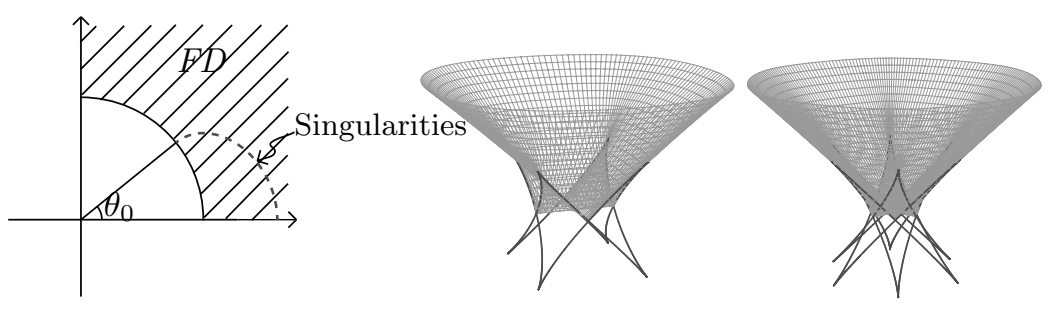

FIgURE 4. Singularities for $X\left(M_{1}\right), X\left(M_{2}\right)$

Lemma 14. Let $r:\left[0, \theta_{0}\right] \rightarrow \mathbb{R}$ be the function defined above.

(i) The image by $X$ of a neighborhood of $\alpha=r(\theta) e^{i \theta}$ for any $\theta \in\left(0, \theta_{0}\right)$ is locally diffeomorphic to a cuspidal edge. The image by $X$ of a neighborhood of $\alpha=r(0)$ is locally diffeomorphic to a swallowtail. The image by $X$ of a neighborhood of $\alpha=e^{i \theta_{0}}$ is locally diffeomorphic to a cuspidal crosscap.

(ii) The singularities form two closed curves in $M_{k}$. In each curve, there are $2(k+1)$ swallowtail singularities. Half of them have constant $t=2 \ln r(0)>$ 0 , and the other half have constant $t=-2 \ln r(0)<0$.

Proof. Let $\hat{\eta}=\frac{\eta}{d \alpha}=1 / \beta$, and ' be the differentiation by $\alpha$. By direct computations, we see that

$$
\frac{g^{\prime}}{g^{2} \hat{\eta}}=\frac{1}{\sigma(k+1)} \frac{\alpha^{2}+1}{\alpha^{2}-1}, \quad \frac{g}{g^{\prime}}=(k+1) \alpha \frac{\alpha^{2}-1}{\alpha^{2}+1}, \quad \frac{g}{g^{\prime}}\left(\frac{g^{\prime}}{g^{2} \hat{\eta}}\right)^{\prime}=\frac{-4}{\sigma} \frac{\alpha^{2}}{\alpha^{4}-1} .
$$

Hence,

$$
\begin{gathered}
\operatorname{Re}\left(\frac{g^{\prime}}{g^{2} \hat{\eta}}\right)=\frac{1}{\sigma(k+1)} \frac{|\alpha|^{4}-1}{\left|\alpha^{2}-1\right|^{2}}, \quad \operatorname{Im}\left(\frac{g^{\prime}}{g^{2} \hat{\eta}}\right)=\frac{-i}{\sigma(k+1)} \frac{\bar{\alpha}^{2}-\alpha^{2}}{\left|\alpha^{2}-1\right|^{2}} \\
\operatorname{Re}\left\{\frac{g}{g^{\prime}}\left(\frac{g^{\prime}}{g^{2} \hat{\eta}}\right)^{\prime}\right\}=\frac{-2}{\sigma}\left(\frac{\alpha^{2}}{\alpha^{4}-1}+\frac{\bar{\alpha}^{2}}{\bar{\alpha}^{4}-1}\right), \\
\operatorname{Im}\left\{\frac{g}{g^{\prime}}\left(\frac{g^{\prime}}{g^{2} \hat{\eta}}\right)^{\prime}\right\}=\frac{2 i}{\sigma}\left(\frac{\alpha^{2}}{\alpha^{4}-1}-\frac{\bar{\alpha}^{2}}{\bar{\alpha}^{4}-1}\right) .
\end{gathered}
$$

Now we apply the criteria in [17, Theorem 3.1] and [8, Theorem 2.3] to conclude (i). (ii) is obvious from Lemma 10 .

6.5. Horizontal cross sections of $X\left(M_{k}\right)$. The horizontal cross sections of the maximal surfaces are obtained as the image of the circles $|\alpha|=$ constant because of (5) (Figure 5). $(\alpha, \beta)=(0,0)$ is the bottom end, and $(\alpha, \beta)=(\infty, \infty)$ is the top end since $\lim _{\alpha \rightarrow 0} t(\alpha, \beta)=-\infty$ and $\lim _{\alpha \rightarrow \infty} t(\alpha, \beta)=\infty$.

6.6. About the orientation. We see that $g(0,0)=g(\infty, \infty)=\infty$, which means that both of the normals at the top and at the bottom catenoidal ends are past pointing (or future pointing depending upon our choice). Note that the normals at the two ends of the elliptic catenoids are in opposite directions. This is in complete analogy with the relation between Costa-Hoffman-Meeks surfaces and catenoids in Euclidean three-space. 

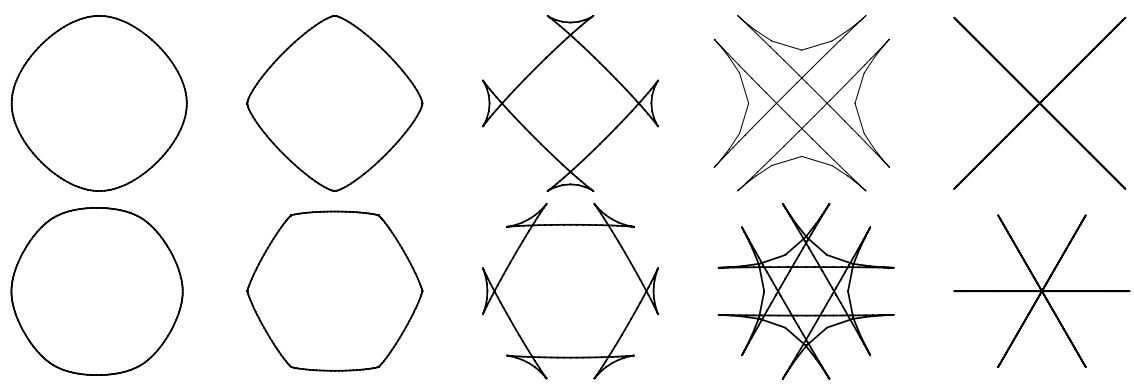

Figure 5. Horizontal cross sections of $X\left(M_{1}\right), X\left(M_{2}\right)$ as $t \rightarrow 0^{+}$

6.7. The symmetry group of $X\left(M_{k}\right)$. Having understood the geometry of $X\left(M_{k}\right)$, we can now determine the full symmetry group of $X\left(M_{k}\right)$.

Lemma 15. The symmetry group $G$ of $X\left(M_{k}\right)$ is of order $8(k+1)$, and is generated by $K, L, M$.

Proof. Note that $g(0,0)=g(\infty, \infty)=\infty$. Since a symmetry sends ends to ends, it preserves vertical normals. On $X\left(M_{k}\right)$, we see that $X(1,0)$ and $X(-1,0)$ are the only points where the Gauss map is vertical. Since both of them are mapped to $\overrightarrow{0} \in \mathbb{L}^{3}$ by $X$, any element of $G$ must fix $\overrightarrow{0}$ and leave the $t$-axis invariant.

Let $G_{1}$ be the subgroup of $G$ of time orientation-preserving isometries. Then the index $\left[G: G_{1}\right]$ of $G_{1}$ in $G$ is 2 . Let $G_{2}$ be the subgroup of $G_{1}$ of orientationpreserving isometries. Then $\left[G_{1}: G_{2}\right]=2$ and $G_{2}$ is a cyclic rotation group around the $t$-axis. The order of $G_{2}$ is at least $2(k+1)$ since it contains the rotation by $\frac{\pi}{k+1}$. On the other hand, since any element in $G_{2}$ must send a swallowtail singularity to another swallowtail singularity of the same time value, and since there are no or $2(k+1)$ swallowtail singularities on horizontal planes, the order of $G_{2}$ is $2(k+1)$.

Combining them all, we see that $G$ is of order $8(k+1)$. Since $G$ contains the group generated by $K, L, M$ which is of order $8(k+1)$, we get the conclusion.

\section{Appendix A. Singly Periodic maximal And minimal Surfaces}

Integrating the Weierstrass data (4) with $k=0$ and with $\alpha=i$ as the reference point of integration yields the null holomorphic curve $\left(\Psi_{1}, \Psi_{2}, \Psi_{0}\right)$ in $\mathbb{C}_{1}^{3}$ after $\alpha$ is substituted by $\frac{w+i}{w-i}$, while the null holomorphic curve $\left(\Phi_{1}, \Phi_{2}, \Phi_{3}\right)$ in $\mathbb{C}^{3}$ is obtained by chance:

$$
\begin{aligned}
\Psi_{1} & =-\frac{1}{2} \ln w+2 \sigma^{2} \frac{w^{2}-1}{w^{2}+1}, & \Phi_{1} & =-\frac{1}{2} \ln w-2 \sigma^{2} \frac{w^{2}-1}{w^{2}+1} \\
\Psi_{2} & =-\frac{i}{2} \ln w-2 i \sigma^{2} \frac{w^{2}-1}{w^{2}+1}, & \Phi_{2} & =-\frac{i}{2} \ln w+2 i \sigma^{2} \frac{w^{2}-1}{w^{2}+1} \\
\Psi_{0} & =2 \sigma \ln \left(-i \frac{w+i}{w-i}\right), & \Phi_{3} & =2 \sigma \ln \left(-i \frac{w+i}{w-i}\right) .
\end{aligned}
$$

So, for any nonzero real number $\sigma$, the real parts of $\left(\Psi_{1}, \Psi_{2}, \Psi_{0}\right)$ and $\left(\Phi_{1}, \Phi_{2}, \Phi_{3}\right)$ give maximal and minimal surfaces, respectively, well defined on the universal cover 

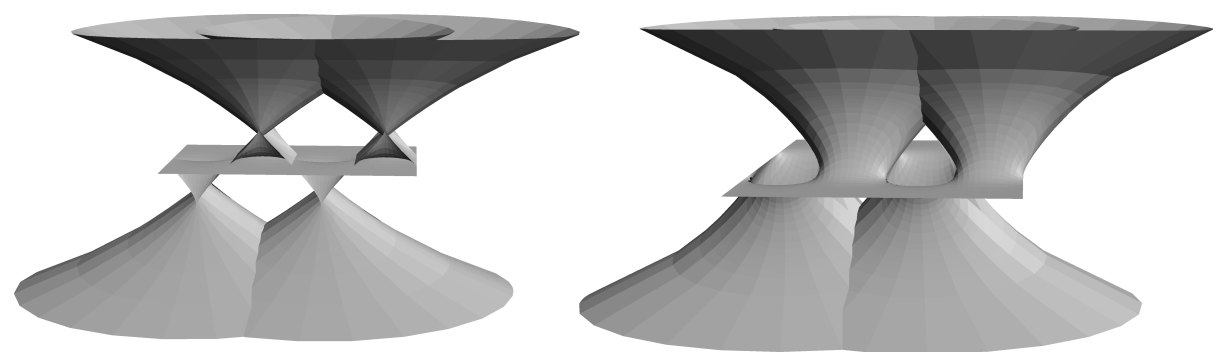

FiguRE 6. Singly periodic maximal and minimal surfaces

of $\hat{\mathbb{C}} \backslash\{0, \infty, i,-i\} .0, \infty$ are planar ends, and $i,-i$ are catenoidal ends. They are singly periodic (Figure 6).

The Weierstrass data (4) for $k \geq 1$ produces doubly periodic minimal surfaces in $\mathbb{E}^{3}$.

\section{REFERENCES}

[1] Luis J. Alías, Rosa M. B. Chaves, and Pablo Mira, Björling problem for maximal surfaces in Lorentz-Minkowski space, Math. Proc. Camb. Phil. Soc., 134 (2003), 289-316. MR.1972140 (2004d:53076)

[2] E. Calabi, Examples of Bernstein problems for some nonlinear equations, Proc. Symp. Pure Math., 15 (1970), 223-230. MR0264210 (41:8806)

[3] S.-Y. Cheng and S.-T. Yau, Maximal space-like hypersurfaces in the Lorentz-Minkowski spaces, Ann. of Math., 104 (1976), 407-419. MR0431061 (55:4063)

[4] F. J. M. Estudillo and A. Romero, Generalized maximal surfaces in Lorentz-Minkowski space $\mathbb{L}^{3}$, Math. Proc. Camb. Phil. Soc., 111 (1992), 515-524. MR1151327 (93b:53010)

[5] I. Fernández and F. López, Periodic maximal surfaces in the Lorentz-Minkowski space $\mathbb{L}^{3}$, arXiv:math.DG/0412461 v3.

[6] I. Fernández, F. López and R. Souam, The space of complete embedded maximal surfaces with isolated singularities in the 3-dimensional Lorentz-Minkowski space $\mathbb{L}^{3}$, arXiv:math.DG/0311330 v2.

[7] I. Fernández, F. López and R. Souam, The moduli space of embedded singly periodic maximal surfaces with isolated singularities in the Lorentz-Minkowski space $\mathbb{L}^{3}$, arXiv:math.DG/0412190 v1.

[8] S. Fujimori, K. Saji, M. Umehara, K. Yamada, Cuspidal crosscaps and singularities of maximal surfaces, Preprint.

[9] D. Hoffman and W. H. Meeks III, Embedded minimal surfaces of finite topology, Ann. of Math., 131 (1990), 1-34. MR1038356 (91i:53010)

[10] T. Imaizumi, Maximal surfaces with conelike singularities of finite type, Kobe J. Math., 18 (2001), 51-60. MR.1868796 (2003c:53014)

[11] T. Imaizumi, Maximal surfaces with simple ends, Kyushu J. Math., 58 (2004), 59-70. MR2053719 (2005a:53010)

[12] O. Kobayashi, Maximal surfaces in the 3-dimensional Minkowski space ${ }^{3}$, Tokyo J. Math., 6 (1983), 297-309. MR0732085 (85d:53003)

[13] O. Kobayashi, Maximal surfaces with conelike singularities, J. Math. Soc. Japan, 36 (1984), 609-617. MR0759417 (86d:53008)

[14] F. J. López, R. López, and R. Souam, Maximal surfaces of Riemann type in LorentzMinkowski space $\mathbb{L}^{3}$, Michigan J. of Math., 47 (2000), 469-497. MR1813540 (2002c:53009)

[15] W. Rossman and K. Sato, Constant mean curvature surfaces with two ends in hyperbolic space, Experimental Math., 7, no. 2 (1998), 101-119. MR1677103(2000b:53014)

[16] R. Schoen, Uniqueness, symmetry, and embeddeness of minimal surfaces, J. Diff. Geom., 18 (1983), 731-809. MR0730928 (85f:53011) 
[17] M. Umehara and K. Yamada, Maximal surfaces with singularities in Minkowski space, arXiv:math.DG/0307309 v6; to appear in Hokkaido Mathematical Journal.

[18] S.-D. Yang, Elliptic catenoids in $\mathbb{L}^{3}$ with an arbitrary number of handles, Proceedings of the International Workshop on Integral Systems, Geometry, and Visualization, Nov. 2004, Kyushu University, Fukuoka, Japan.

Department of Mathematics, Korea University, Seoul 136-713, Korea

E-mail address: ywkim@korea.ac.kr

Department of Mathematics, Korea University, Seoul 136-713, Korea

E-mail address: sdyang@korea.ac.kr 